\title{
El consumo de alcohol y el estrés entre estudiantes del segundo año de enfermería
}

\author{
Elena Tam Phun ${ }^{1}$ \\ Claudia Benedita dos Santos ${ }^{2}$
}

El objetivo de este estudio fue identificar el consumo de alcohol y el estrés académico en estudiantes de enfermería. Se trata de una investigación descriptiva, transversal, que se realizó en el año de 2005. En este estudio los cuestionarios Inventario de Estrés Académico y el AUDIT fueron aplicados en 82 estudiantes de una Universidad privada en Lima, Perú. Los resultados mostraron que los estudiantes tenían edad promedio 20,4 años, siendo $92.7 \%$ solteras, $69.5 \%$ no trabajan, $91.5 \%$ no tenían hijos y $86.6 \%$ vivían con sus padres. La sobrecarga académica representó el estresor de mayor prevalencia, y en relación al consumo de alcohol, 48\% consumieron 1 -2 vasos en un día; $56.1 \%$ una vez al mes y $51.2 \%$ más de tres vasos en un día cualquiera; $11.0 \%$ dejaron de cumplir con sus obligaciones. Un tercio relató fumar, beber o comer demasiado. Se concluyó que todas las situaciones generan diversos niveles de estrés, relacionados al factor actividad/tiempo; siendo la preocupación, la mayor respuesta frente al estrés.

Descriptores: Consumo de Bebidas Alcoholicas; Estrés; Estudiantes de enfermería.

\footnotetext{
${ }^{1}$ Profesora Auxiliar, Facultad de Enfermería, Universidad Peruana Cayetano Heredia, Peru. E-mail: etam@upch.edu.pe.

2 Doctor en Estadística, Profesor Asociado, Escola de Enfermagem de Ribeirão Preto, Universidade de São Paulo, Centro Colaborador de la OMS para el Desarrollo de la Investigación en Enfermería, Brasil. E-mail: cbsantos@eerp.usp.br.
}

Correspondencia:

Claudia Benedita dos Santos

Universidade de São Paulo. Escola de Enfermagem de Ribeirão Preto

Av. Bandeirantes, 3900

Bairro Monte Alegre

CEP: 14040-902 Ribeirão Preto, SP, Brasil

E-mail: cbsantos@eerp.usp.br 


\section{Consumo de álcool e estresse em estudantes do segundo ano de enfermagem}

O objetivo deste estudo foi identificar o consumo de álcool e o estresse acadêmico em estudantes de Enfermagem. Como método, usou-se a investigação descritiva, transversal, realizada no ano 2005. Neste estudo, os questionários Inventário de Estresse Acadêmico e AUDIT foram aplicados em 82 estudantes de uma universidade privada em Lima, Peru. Os resultados mostram que os estudantes apresentaram idade média de 20,4 anos, sendo que $92,7 \%$ eram solteiros, $69,5 \%$ não trabalhavam, $91,5 \%$ não tinham filhos e $86,6 \%$ viviam com seus pais. A sobrecarga acadêmica representou o estressor de maior prevalência e, em relação ao consumo de álcool, $48 \%$ consumiam 1-2 copos por dia, $56,1 \%$ uma vez ao mês e $51,2 \%$ mais de três copos em um dia qualquer, e $11 \%$ deixaram de cumprir com suas obrigações. Um terço relatou fumar, beber ou comer demasiadamente. Conclui-se que todas as situações geraram diversos níveis de estresse, relacionados ao fator atividade/tempo, sendo a preocupação a maior resposta frente ao estresse.

Descritores: Consumo de Bebidas Alcoólicas; Estresse; Estudantes de Enfermagem.

\section{Alcohol Consumption and Stress in Second Year Nursing Students}

The aim of this study was to identify the consumption of alcohol and academic stress in nursing students. This was a descriptive, cross-sectional study, performed in 2005. The questionnaires Academic Stress Inventory and AUDIT were applied to 82 students of a private University in Lima, Peru. The students had a mean age of 20.4 years, $92.7 \%$ were single, $69.5 \%$ did not work, $91.5 \%$ had no children and $86.6 \%$ lived with their parents. The academic overload represented the stressor of highest prevalence. Regarding the consumption of alcohol, $56.1 \%$ consumed alcohol once a month, $48.0 \%$ consumed $1-2$ glasses a day, and $51.2 \%$ more than three glasses during a normal day; $11.0 \%$ failed to comply with their obligations. One third reported smoking, drinking or eating in excess. All situations, related to the factor activity/time, generated various levels of stress, with worry reported as the main response to stress.

Descriptors: Alcohol Drinking; Stress; Nursing Students.

\section{Introducción}

Fue por el año 1936 cuando Hans Selye, preocupado con el síndrome de "estar enfermo" inició las investigaciones que lo llevaron a usar la palabra Estrés y el concepto sobre el Síndrome General de Adaptación (SGA o GAS por sus siglas en inglés), tan de moda en nuestro tiempo. Luego Alvin Toffler que escribió sobre el "Shock del Futuro", hoy considerado el "Shock del Presente", la avasalladora enfermedad que la humanidad padece ante el vertiginoso ritmo de vida y los acelerados cambios a los que es cada día más difícil adaptarse ${ }^{(1)}$. La inadecuada adaptación del organismo ante el agente agresor por un tiempo prolongado, baja las defensas y predispone a enfermedades mentales, conflictos y desequilibrios emocionales, los que son reflejados en un $75 \%$ de las consultas médicas por afecciones relacionadas en forma directa o indirecta con el estrés ${ }^{(2)}$. El tema del estrés ha sido enfocado en los diferentes campos del conocimiento durante el siglo $X X$, identificándose dos líneas de investigación: la psicofisiológica y la psicológica, las que son de interés para la presente investigación; siendo escasos los trabajos relacionados al estrés académico entre estudiantes universitarios. En la actualidad han cobrado auge las teorías interaccionistas del estrés que plantean que la respuesta al estrés es el resultado de 
la interacción entre las características de la persona y las demandas del medio. Se considera que una persona está en una situación estresante o bajo un estresor cuando debe hacer frente a situaciones que conllevan demandas conductuales que le resultan difíciles de poner en práctica o de satisfacer ${ }^{(3)}$.

Actualmente, la sociedad atraviesa por un periodo de grandes cambios, originando estrés en las personas, esto se concretiza en los requerimientos señalados por las instituciones u organizaciones en la solicitud de empleo: "se necesita que sepan trabajar bajo presión". Las manifestaciones de estrés, en las personas, se han visto incrementadas como lo señalan las investigaciones realizadas en diferentes países, en su mayor parte orientadas al estudio del estrés en ámbitos laborales, a su repercusión en las personas y a su actuación; en estas investigaciones se ha encontrado que los cambios producidos en las diferentes estructuras de la sociedad, demandan un proceso de adaptación constante del ser humano a las nuevas situaciones originadas por el avance científico y tecnológico y por los efectos de la globalización en todos los campos del desarrollo humano.

Algunos estudios realizados demuestran que las diversas actividades académicas representan una importante fuente de estrés para los estudiantes universitarios $^{(4)}$. El estrés no controlado ante el fracaso de la capacidad de adaptación, producto del acelerado cambio de vida actual, lleva a muchos hacia el bloqueo mental como única forma de evadirse de una realidad que no son capaces de enfrentar, ni comprender(5).

Debido a lo anterior, muchas instituciones como la CICAD-OEA vienen dedicando esfuerzos no sólo para su identificación sino también para plantear y difundir nuevas alternativas - ante los nuevos retos producidos por el consumo de alcohol, drogas y otras sustancias psicoactivas - consideradas de gran importancia para disminuir los daños asociados.

Identificar el consumo de alcohol y el estrés académico en estudiantes de enfermería es el objetivo del presente estudio.

\section{Importancia del Estudio}

El cuidado integral de la persona sana o enferma demanda una preparación académica esmerada desde diversos ángulos del saber científico y tecnológico (biológico, psicológico, social, económico y político, entre otros); sin embargo este cuidado integral del estudiante pasa desapercibido en los planes de estudios de las instituciones formadoras, inclusive en el de los de profesionales de enfermería, lo que se hace evidente en los problemas de salud que frecuentemente presentan, quizás, originados por estrés originado por los cortos periodos de aprendizaje, sobre todo en lo que se refiere a la obtención de experiencias clínicas de enfermería, en ambientes sobrecargados de estudiantes, provenientes de diversas instituciones y niveles de formación profesional, tratando de competir, muchas veces de manera desigual, por las mismas experiencias; esto debido a las escasas oportunidades de entrenamiento y experiencia de aprendizaje en relación al número de pacientes y al número de estudiantes.

\section{Metodología}

El diseño de investigación descriptiva, transversal, se realizó en el año de 2005. La población de estudio estuvo constituida por el total de estudiantes matriculadas en el segundo año del programa de enfermería pre grado del turno de la mañana, en Universidad Peruana Cayetano Heredia, Facultad de Enfermería, en Lima, en 2005, siendo 89 estudiantes según el registro de matrícula, que reunían los criterios de inclusión y exclusión. Quedando conformada la muestra por 82 estudiantes que respondieron el cuestionario después de haber sido informados y consentido en participar; cinco no respondieron a todas las preguntas $y$ dos estuvieron ausentes.

Técnica e Instrumentos - La recolección de la información se realizó a través de un cuestionario autoadministrado que comprendió: Aspectos sociodemográficos y nivel de rendimiento actual. El Inventario de Estrés $(\mathrm{IEA})^{(6)}$ fue diseñado específicamente para la evaluación del estrés académico en universitarios; este tiene capacidad discriminativa para la detección de diferentes situaciones académicas (11 ítems); para cada una de las situaciones planteadas presenta una escala con valores de 1 a 5 (1 representa Nada de estrés y 5 Mucho estrés) que indican el grado de estrés que dicha situación puede generar; $y$, las respuestas experimentadas ante tales situaciones (11 ítems) con aspectos cognitivos (ítems 1, 4, 7, 10 y 12), fisiológicos (ítems 2, 5, 8 y 11) o motores (ítems 3, 6 y 9). La frecuencia de ocurrencia de las respuestas, referidas a los tres niveles de respuesta del organismo, se evalúan en una escala de 1 a 5 , donde 1 significa Nunca e 5 significa Siempre; El Test para medir el consumo de alcohol AUDIT (Alcohol use Disorders Identification Test), también identificado como Test de 
Identificación de los Trastornos Debidos al Consumo de Alcohol, es un instrumento de evaluación desarrollado y propuesto por la Organización Mundial de la Salud (OMS) como un método simple para evaluar el consumo de alcohol en el ámbito de la atención primaria en el ámbito internacional, ayuda a identificar si la persona presenta un consumo de riesgo, un consumo perjudicial o una dependencia de alcohol(3,7). El test AUDIT ha sido validado en pacientes de atención primaria en seis países: Noruega, Australia, Kenia, Bulgaria, México y los Estados Unidos de América(3); posteriormente fue validado para el españo|(7). La literatura internacional señala la validación de AUDIT, con una sensibilidad de $92 \%$ y una especificidad de $93 \%$; es un test que puede ser aplicado con rapidez ${ }^{(8)}$

Se realizó una prueba piloto con diez estudiantes que no formaron parte de la muestra, para reajustar los aspectos de semántica, lo cual no fue necesario, ya que las estudiantes no mostraron dificultad para responder a las preguntas del instrumento. La aplicación de los instrumentos se realizó de manera colectiva en la misma sala de clases, previa coordinación con la Institución educativa y con los profesores responsables para el permiso correspondiente. En el día programado se invitó a las estudiantes a participar en el estudio, se les informó sobre el estudio, los objetivos, la finalidad - después de las informaciones se firmó el término de consentimiento como requisito para participar.

\section{Resultados}

Se revisaron 82 cuestionarios respondidos de manera completa por las estudiantes que en su totalidad eran del sexo femenino. En la Tabla 1 se puede observar el perfil de la estudiante de enfermería que conformó la muestra de estudio.
Profundizando el análisis se observan resultados que por su naturaleza son importantes al estar relacionados con el factor tiempo/actividad, a pesar de haber sido informados por un porcentaje bajo de estudiantes: tienen hijos, trabaja, es casada, viven con la pareja u otro familiar.

Tabla 1 - Perfil de las estudiantes de Pregrado de la Universidad Peruana Cayetano Heredia. Facultad de Enfermería. Lima 2005

\begin{tabular}{|c|c|c|}
\hline Total & $n^{\circ}$ & $\%$ \\
\hline \multicolumn{3}{|l|}{ Grupo etario (años) } \\
\hline $16-17$ & 03 & 3.7 \\
\hline $18-19$ & 39 & 47.5 \\
\hline $20-21$ & 19 & 23.2 \\
\hline $22-23$ & 13 & 15.8 \\
\hline 24 o más & 08 & 9.8 \\
\hline \multicolumn{3}{|c|}{ Edad: $\bar{\chi}=20.4 ; M d=19$ años $D S=0.7$} \\
\hline \multicolumn{3}{|l|}{ Estado Civil } \\
\hline Soltera & 76 & 92.7 \\
\hline Casada & 06 & 7.3 \\
\hline \multicolumn{3}{|l|}{ Tiene Hijos } \\
\hline No & 75 & 91.5 \\
\hline $\mathrm{Si}$ & 07 & 8.5 \\
\hline \multicolumn{3}{|l|}{ Trabaja } \\
\hline No & 57 & 69.5 \\
\hline $\mathrm{Si}$ & 25 & 30.5 \\
\hline \multicolumn{3}{|l|}{ Con quien vive } \\
\hline Padres & 71 & 86.6 \\
\hline Pareja & 04 & 4.9 \\
\hline Otros & 07 & 8.5 \\
\hline \multicolumn{3}{|c|}{ Promedio asignaturas generales (semestre anterior) } \\
\hline $11-13$ & 52 & 63.4 \\
\hline $14-17$ & 30 & 36.6 \\
\hline \multicolumn{3}{|c|}{ Promedio asignaturas Enfermería (Semestre anterior) } \\
\hline $11-13$ & 59 & 72.0 \\
\hline $14-17$ & 23 & 28.0 \\
\hline
\end{tabular}

En la Tabla 2 se aprecia el nivel de estrés relacionado a cada una de las situaciones consideradas en el inventario de Estrés Académico IEA.

Tabla 2 - Análisis descriptivo del grado de estrés generado por cada una de las situaciones (IEA). Estudiantes de Pregrado de la Universidad Peruana Cayetano Heredia. Facultad de Enfermería. Lima 2005

\begin{tabular}{|c|c|c|c|c|c|c|c|c|c|c|}
\hline \multirow{2}{*}{$\begin{array}{l}\text { Puntajes* }^{*} \\
\text { Situación }\end{array}$} & \multicolumn{2}{|c|}{1} & \multicolumn{2}{|c|}{2} & \multicolumn{2}{|c|}{3} & \multicolumn{2}{|c|}{4} & \multicolumn{2}{|c|}{5} \\
\hline & $n^{\circ}$ & $\%$ & $n^{\circ}$ & $\%$ & $n^{\circ}$ & $\%$ & $n^{\circ}$ & $\%$ & $n^{\circ}$ & $\%$ \\
\hline 1. Realización de un examen & 2 & 2.4 & 8 & 9.8 & 31 & 37.8 & 26 & 31.7 & 15 & 18.3 \\
\hline 2. Exposición de trabajos en clase & 4 & 4.9 & 17 & 20.7 & 28 & 34.1 & 27 & 32.9 & 6 & 7.3 \\
\hline $\begin{array}{l}\text { 3. Intervención en la clase (responder a una pregunta del } \\
\text { profesor, realizar preguntas, participar en coloquios, etc.) }\end{array}$ & 5 & 6.1 & 24 & 29.3 & 28 & 34.1 & 22 & 26.8 & 3 & 3.7 \\
\hline 4. Subir al despacho del profesor en horas de tutoría & 18 & 21.9 & 30 & 36.6 & 22 & 26.8 & 9 & 11.0 & 3 & 3.7 \\
\hline $\begin{array}{l}\text { 5. Sobrecarga académica (excesivo número de créditos, } \\
\text { trabajos obligatorios, etc.) }\end{array}$ & 0 & 0 & 5 & 6.1 & 26 & 31.7 & 25 & 30.5 & 26 & 31.7 \\
\hline 6. Masificación de las aulas & 9 & 11.0 & 12 & 14.6 & 35 & 42.7 & 17 & 20.7 & 9 & 11.0 \\
\hline
\end{tabular}


Tabla 2 - Continuación

\begin{tabular}{|c|c|c|c|c|c|c|c|c|c|c|}
\hline \multirow{2}{*}{$\begin{array}{l}\text { Puntajes* } \\
\text { Situación }\end{array}$} & \multicolumn{2}{|c|}{1} & \multicolumn{2}{|c|}{2} & \multicolumn{2}{|c|}{3} & \multicolumn{2}{|c|}{4} & \multicolumn{2}{|c|}{5} \\
\hline & $\mathrm{n}^{\circ}$ & $\%$ & $\mathrm{n}^{\circ}$ & $\%$ & $\mathrm{n}^{\circ}$ & $\%$ & $n^{\circ}$ & $\%$ & $n^{\circ}$ & $\%$ \\
\hline $\begin{array}{l}\text { 7. Falta de tiempo para poder cumplir con las } \\
\text { actividades académicas }\end{array}$ & 1 & 1.2 & 12 & 14.6 & 29 & 35.4 & 24 & 29.3 & 16 & 19.5 \\
\hline 8. Competitividad entre compañeros & 4 & 4.9 & 27 & 32.9 & 29 & 35.4 & 19 & 23.2 & 3 & 3.7 \\
\hline $\begin{array}{l}\text { 9. Realización de trabajos obligatorios para aprobar la } \\
\text { asignatura (búsqueda de material necesario, redactar el } \\
\text { trabajo, etc.) }\end{array}$ & 6 & 7.3 & 11 & 13.4 & 32 & 39.0 & 19 & 23.2 & 14 & 17.1 \\
\hline 10. La tarea de estudio & 5 & 6.1 & 27 & 32.9 & 29 & 35.4 & 16 & 19.5 & 5 & 6.1 \\
\hline 11. Trabajar en grupo & 15 & 18.3 & 33 & 40.2 & 23 & 28.0 & 7 & 8.5 & 4 & 4.9 \\
\hline
\end{tabular}

* Puntajes: 1 representa Nada de estrés y 5 representa Mucho estrés.

Realizando un análisis más profundo, se puede observar que la sobrecarga académica representó el estresor de mayor prevalencia. Subir al despacho del profesor en horas de tutorías y trabajar en grupo representa menor estrés para la estudiante, las otras situaciones representan mediano estrés.

La realización de un examen, la masificación de las clases, la tarea de estudio y las situaciones que requieren establecer una relación interpersonal representan una presión por encima del punto medio para el estudiante; esta es menor cuando se trata de subir al despacho del profesor en horas de tutoría o realizar un trabajo en grupo, situaciones no menos importantes para generar cierto grado de estrés en la estudiante.

La Tabla 3 indica elementos donde se recoge información en torno a los tres niveles de respuesta, fisiológico, cognitivo y motor, que el organismo experimenta cuando se ve expuesto a situaciones de estrés.

Tabla 3 - Análisis descriptivo de los tres niveles de respuesta del organismo ante el Estrés generado por cada situación (IEA) en estudiantes de Pregrado en la Universidad Peruana Cayetano Heredia. Facultad de Enfermería. Lima 2005

\begin{tabular}{|c|c|c|c|c|c|c|c|c|c|c|}
\hline \multirow{2}{*}{$\begin{array}{c}\text { Puntajes* } \\
\text { Respuestas }\end{array}$} & \multicolumn{2}{|c|}{1} & \multicolumn{2}{|c|}{2} & \multicolumn{2}{|c|}{3} & \multicolumn{2}{|c|}{4} & \multicolumn{2}{|c|}{5} \\
\hline & $n^{\circ}$ & $\%$ & $n^{\circ}$ & $\%$ & $n^{\circ}$ & $\%$ & $n^{\circ}$ & $\%$ & $n^{\circ}$ & $\%$ \\
\hline 1. Me preocupo & 5 & 6.1 & 13 & 15.9 & 23 & 28.0 & 17 & 20.7 & 24 & 29.3 \\
\hline $\begin{array}{l}\text { 2. El corazón me late muy rápido y/o } \\
\text { me falta aire y la respiración es agitada }\end{array}$ & 17 & 20.7 & 23 & 28.0 & 29 & 35.4 & 6 & 7.3 & 7 & 8.5 \\
\hline $\begin{array}{l}\text { 3. Realizo movimientos repetitivos } \\
\text { con alguna parte de mi cuerpo, me } \\
\text { quedo paralizado o mis movimientos } \\
\text { son torpes }\end{array}$ & 22 & 26.8 & 24 & 29.3 & 22 & 26.8 & 10 & 12.2 & 4 & 4.9 \\
\hline 4. Siento miedo & 8 & 9.8 & 28 & 34.1 & 28 & 34.1 & 11 & 13.4 & 7 & 8.5 \\
\hline 5. Siento molestias en el estómago & 17 & 20.7 & 23 & 28.0 & 24 & 29.3 & 11 & 13.4 & 7 & 8.5 \\
\hline 6 Fumo, como o bebo demasiado & 55 & 67.1 & 8 & 9.8 & 12 & 14.6 & 4 & 4.9 & 3 & 3.7 \\
\hline $\begin{array}{l}\text { 7. Tengo pensamientos o sentimientos } \\
\text { negativos }\end{array}$ & 35 & 42.7 & 20 & 24.4 & 16 & 19.5 & 8 & 9.8 & 3 & 3.7 \\
\hline 8. Me tiemblan las manos o las piernas & 27 & 32.9 & 21 & 25.6 & 16 & 19.5 & 8 & 9.8 & 10 & 12.2 \\
\hline $\begin{array}{l}\text { 9. Me cuesta expresarme verbalmente } \\
\text { o a veces tartamudeo }\end{array}$ & 29 & 35.4 & 20 & 24.4 & 17 & 20.7 & 9 & 11.0 & 7 & 8.5 \\
\hline 10. Me siento inseguro de mi mismo & 30 & 36.6 & 22 & 26.8 & 20 & 24.4 & 4 & 4.9 & 6 & 7.3 \\
\hline $\begin{array}{l}\text { 11. Se me seca la boca y tengo } \\
\text { dificultades para tragar }\end{array}$ & 46 & 56.1 & 16 & 19.5 & 12 & 14.6 & 2 & 2.4 & 6 & 7.3 \\
\hline 12. Siento ganas de llorar & 38 & 46.3 & 23 & 28.0 & 12 & 14.6 & 3 & 3.7 & 6 & 7.3 \\
\hline
\end{tabular}

*Puntajes - 1 representa Nunca y 5 representa Siempre.

Se aprecia que el nivel cognitivo (ítems $1,4,7,10$ y 12 ) presentó mayor promedio, con $\bar{\chi}=2,5$; de $=1,3 \mathrm{e}$ $\mathrm{md}=2$, seguido de fisiológico (ítems 2, 5, 8 y 11), con $\bar{\chi}=2,4 ;$ de $=1,2$ e $\mathrm{md}=2$ y motor (ítems 3, 6 y 9), con $\bar{\chi}=2,1$; de $=1,2$ e $\mathrm{md}=2$ ).

En la Tabla 4 se observa el Consumo de Alcohol de las estudiantes, según el teste AUDIT. 
Tabla 4 - Consumo de Alcohol de estudiantes Pregrado (AUDIT) de la Universidad Peruana Cayetano Heredia. Facultad de Enfermería. Lima 2005

1 ¿Con qué frecuencia consume alguna bebida alcohólica?

(0) Nunca (Pase a las preguntas 9-10)

(1) Una o menos veces al mes

(2) De 2 a 4 veces al mes

(3) De 2 a 3 veces a la semana

(4) 4 o más veces a la semana

$\begin{array}{cc}\mathrm{n}^{\circ} & \% \\ 21 & 25.6 \\ 46 & 56.1 \\ 15 & 18.3 \\ 0 & 0 \\ 0 & 0 \\ & \\ 40 & 48.8 \\ 11 & 13.4 \\ 10 & 12.2 \\ 7 & 8.5 \\ 14 & 17.1 \\ & \\ 39 & 47.6 \\ 24 & 29.3 \\ 14 & 17.1 \\ 5 & 6.1 \\ 0 & 0\end{array}$

Cuantas consumiciones de bebidas alcohólicas suele realizar en un día de consumo normal?
(0) $1 \circ 2$
(1) $3 \circ 4$
(2) $5 \circ 6$
(3) $7,8,09$
(3) 10 o más

Pase a las preguntas 9 y 10 sí la suma total de las preguntas 2 y $3=0$

4 ¿Con qué frecuencia, en el curso del último año, ha sido incapaz de parar de beber una vez que había empezado?
(0) Nunca
(1) Menos de una vez al mes
(2) Mensualmente
(3) Semanalmente
(4) A diario o casi a diario

5 ¿Con qué frecuencia, en el curso del último año, no pudo hacer lo que se esperaba de usted porque había bebido?
(0) Nunca
(1) Menos de una vez al mes
(2) Mensualmente
(3) Semanalmente
(4) A diario o casi a diario

$\begin{array}{cc}73 & 89.0 \\ 8 & 9.8 \\ 1 & 1.2 \\ 0 & 0 \\ 0 & 0 \\ & \\ 73 & 89.0 \\ 6 & 7.3 \\ 1 & 1.2 \\ 2 & 2.4 \\ 0 & 0\end{array}$

6 ¿Con qué frecuencia, en el curso del último año, ha necesitado beber en ayunas para recuperarse después de haber bebido mucho el día anterior?
(0) Nunca
(1) Menos de una vez al mes
(2) Mensualmente
(3) Semanalmente
(4) A diario o casi a diario

7 ¿Con qué frecuencia, en el curso del último año, ha tenido remordimientos o sentimientos de culpa después de haber bebido?

(0) Nunca

(1) Menos de una vez al mes

(2) Mensualmente

(3) Semanalmente

(4) A diario o casi a diario

8 ¿Con qué frecuencia, en el curso del último año, no ha podido recordar lo que sucedió la noche anterior porque había estado bebiendo?

(0) Nunca

(1) Menos de una vez al mes

(2) Mensualmente

(3) Semanalmente

(4) A diario o casi a diario

9 ¿Usted o alguna otra persona ha resultado herida porque usted había bebido?
(0) No
(2) $\mathrm{Si}$, pero no en el curso del ultimo año

(4) Si, en el último año

0 ¿Algún familiar, amigo, médico o profesional sanitario ha mostrado preocupación por su consumo de bebidas alcohólicas o le han sugerido que deje de beber?
(0) No
(2) Si, pero no en el curso del ultimo año 


\section{Discusión}

La profesión de enfermería es una carrera realizada principalmente por mujeres, el porcentaje de varones es mínimo o a veces ausente en algunos grupos, como en la población del presente estudio que encontrándose dos varones, estos no llegaron a participar por no estar presentes en la recolección de datos. Los estudios de enfermería se caracterizan por una exigencia académica creciente que demanda de la estudiante de enfermería esfuerzos permanentes de adaptación frente a la demanda académica tanto teórica como práctica; a esto se agrega la necesidad de obtener una preparación de óptima calidad, fundamental para desempeñarse profesionalmente en un mercado cada vez más competitivo; además, debe tenerse presente que la carrera se desarrolla en la adolescencia y la adultez joven, ciclos vitales muy especiales por las características que poseen. Lo anterior presupone un nivel alto de estrés en la estudiante de enfermería en el transcurso de su formación académica, con riesgo de alteración en su salud física y mental (Tabla 1). En un estudio realizado por Sender y col. en 32 estudiantes de medicina que solicitaron ayuda para enfrentar el miedo a los exámenes, $90 \%$ de los sujetos eran mujeres y el $25 \%$ recibieron o estaban recibiendo atención psicológica o psiquiátrica(9). El mayor porcentaje de estudiantes que participaron en el estudio viven con la familia nuclear y no trabajan, es conocida la función homeostática de la familia como soporte social frente a la influencia nociva del estrés. En nuestra cultura gran parte de este soporte depende de las relaciones familiares. El soporte social es definido por Cobb, como "información que conduce a creer que uno es cuidado, amado y estimado". Se ha encontrado suficiente evidencia para aceptar que ese soporte representa un factor protector inespecífico opuesto a la acción nociva del estrés, lo que beneficia la salud y favorece la recuperación de la enfermedad(9-10). Asimismo la relación de pareja constituye un importante soporte social en la etapa del ciclo vital de las estudiantes de enfermería.

Los resultados del presente estudio muestran un porcentaje relativamente importante de estudiantes que refieren presentar un nivel medio y alto de estrés académico; así mismo todas expresan presentar estrés en algún nivel frente a las situaciones planteadas en el cuestionario. En relación a las respuestas frente a las situaciones de estrés estas son expresadas en niveles por debajo del punto medio en una escala de 5 .
No habiéndose encontrado estudios realizados en estudiantes de enfermería se recurre a contrastar los resultados del presente estudio con investigaciones realizadas en estudiantes de medicina por tratarse de estudiantes que se ven expuestos a situaciones académicas similares, tales como intensas horas de estudio, masificación de las aulas, realización de trabajos obligatorios, entre otras situaciones académicas potencialmente estresantes. Muchos de los autores(11) refieren que la carrera de medicina es muy estresante desde el inicio de la carrera y demanda de las Facultades de Medicina una mayor atención en el cuidado de la salud mental de sus estudiantes. Las respuestas ante el estrés del estudio realizado son similares a los encontrados en estudio publicado en 1996 que refieren hallazgos de puntuación media(6).

En relación al consumo de alcohol en las estudiantes de enfermería que constituyeron la muestra, los resultados del estudio nos muestra cifras por encima del $50 \%$ (Tabla 4). Perales C.A., en su estudio en estudiantes, de dos universidades peruanas, encontró que la prevalencia de vida del consumo de alcohol en ambas universidades era de $95.6 \%$ y $86.8 \%$, siendo la prevalencia de vida de abuso $57.8 \%$ y $55.7 \%$. Coincidiendo también a los datos obtenidos en encuestas nacionales una prevalencia de vida de uso de $61 \%$ entre los jóvenes de 15 a 17 años y $89 \%$ entre los 21 a 24 años, por lo que se puede observar que el consumo de alcohol sigue el patrón de consumo de nuestra cultura; vinculado a cualquier situación relacionada a un triunfo, a una derrota, un motivo de alegría o de tristeza(11).

\section{Conclusiones}

Todas las estudiantes de enfermería del segundo año, que participaron en el estudio, presentaron diversos niveles de estrés. En la sobrecarga académica: excesivo número de créditos y trabajos obligatorios fueron las situaciones que presentaron el estresor de mayor prevalencia. La respuesta frente al estrés fue: fumo, bebo o como demasiado, fue expresado por casi un tercio de la muestra de estudiantes que participaron en el estudio. El uso y prevalencia de alcohol se encuentra por encima del $50 \%$.

\section{Recomendaciones}

A los responsables de los programas de formación universitaria se les recomienda establecer programas preventivos para promover el uso de hábitos saludables y 
disminuir hábitos dañinos (consumo de alcohol y drogas psicoactivas) para la salud y el bienestar; también deben disminuir las conductas de riesgo y el riesgo de morbilidad y mortalidad.

\section{Agradecimientos}

Agradecemos a la Comisión Interamericana para el Control del Abuso de Drogas/CICAD de la Secretaria de Seguridad Multidimensional/SSM de la Organización de los Estados Americanos/OEA, la Secretaria Nacional de
Políticas sobre Drogas/SENAD do Gabinete de Seguridad Institucional/Brasil, la Escuela de Enfermería de Ribeirao Preto de la Universidad de Sao Paulo y Centro Colaborador de la Organización Mundial de la Salud para el Desarrollo de la Investigación en Enfermería, la población representada en los estudios de investigación, bien como a las autoridades de las universidades representadas por los participantes del Programa En-Line de Especialización en Investigación sobre el Fenómeno de las Drogas - PREINVEST, periodos 2005, 2006, 2007 y 2008.

\section{Referencias}

1. Buendía J. Psicología Médica. Madrid: Pirámide; 2002.

2. Huaquin M, Víctor R, Loaiza H. Exigencias Académicas y Estrés en las Carreras de la Facultad de Medicina de la Universidad Austral de Chile. [Estud. Pedagógico on line] 2004, [citado 2005 Mayo 02]; 30. Disponible en: http://www.scielo.cl/scielo. phpp?script=sci_arttext\&pid.

3. Seperiza P. CiberEscrito No 6 de Mundo Mejor. Chile: Quilpue; 1994. Disponible en: http://www.monografias.com/trabajos14/ estres/estres.shtml

4. Burgos G, María F. Calidad de Vida y Estrés de los Estudiantes de la Escuela de Enfermería, Universidad Austral de Chile, 2 Semestre Académico 2003. [on line] 2004, [citado 2004 Jan 01]. Universidad Austral de Chile. Université Lyon 2 - ERAD. Disponible en: http://cybertesis.uach.cl/tesis/uach/2004/ fmb957c/html/index -frames.html

5. Babor TF, Higgins-Biddle JC. AUDIT. Washington: Organización Mundial de la Salud; 2001.

6. Polo A, Hernández J.M, Pozo C. Evaluación del Estrés Académico en Estudiantes Universitarios. Rev. Ansiedad y Estrés $1996 ; 2(2 / 3): 159-72$.
7. Montero SA, Casado PG CP, La Cruz CL, Fernández FB. Papel del test AUDIT (Alcohol Use Disorders Identification Test) para la detección de consumo excesivo de alcohol. Medifam 2001; 11(9):83-7.

8. Gonçalves JRL, O Uso de Bebidas Alcoólicas entre os Cortadores de Cana de Uma Agroindústria Sucroalcooleira. [Dissertação]. Ribeirão Preto (SP): Escola de Enfermagem de Riberão Preto/ USP; 2000.

9. Sender R, Valles A, Puig O, Salamero M, Valdés $M$. ¿Qué hay detrás del miedo a los exámenes? Educ. Méd. 2004; 7(1):36-41. 10. Gil HLB, Mello DF, Ferriani MGC, Silva MAI. Perceptions of adolescents students on the consumption of drugs: a case study in Lima, Peru. Rev. Latino-Am. Enfermagem. 2008, 16 (Spe):551-7.

11. Perales A, Sogi C, Morales R. Estudio comparativo de salud mental en estudiantes de medicina de dos universidades estatales peruanas. Anales Fac. Med. 2003. Universidad Nacional Mayor de San Marcos; 64(4): 236-46. 